\title{
BPs Operational Matrices for Solving Time Varying Fractional Optimal Control Problems
}

\author{
Mohsen Alipour ${ }^{1}$, Davood Rostamy ${ }^{1}$ \\ ${ }^{1}$ Department of Mathematics, Imam Khomeini International University, \\ P.O. Box 34149-16818, Qazvin, Iran \\ m.alipour2323@gmail.com; rostamy@khayam.ut.ac.ir
}

Article history:

Received January 2013

Accepted February 2013

Available online April 2013

\begin{abstract}
In this paper, we present a method for solving time varying fractional optimal control problems by Bernstein polynomials. Firstly, we derive the Bernstein polynomials (BPs) operational matrix for the fractional derivative in the Caputo sense, which has not been undertaken before. This method reduces the problems to a system of algebraic equations. The results obtained are in good agreement with the existing ones in open literatures and the solutions approach to classical solutions as the order of the fractional derivatives approach to 1 .
\end{abstract}

Keywords: Time varying fractional optimal control problems, Bernstein polynomials, operational matrix, Caputo derivative.

\section{Introduction}

Although the optimal control theory is an area in mathematics which has been under development for years but the fractional optimal control theory is a very new area in mathematics. Recent contributions in this field were reported by several authors [1-4].

In this paper, we consider the time varying fractional optimal control problem as follows:

Minimize $\quad J(x(t), u(t))=\frac{1}{2} \int_{0}^{1} x^{2}(t)+u^{2}(t) d t$,

subject to the dynamic constrain 


$$
D^{\alpha} x(t)=a_{1}(t) x(t)+a_{2}(t) u(t), \quad 0<t \leq 1,0<\alpha \leq 1,
$$

and the initial condition

$x(0)=x_{0}$,

where $x(t)$ and $u(t)$ are the state function and the control function, respectively. When $\alpha=1$, the above problem reduces to a standard optimal control problem.

The rest of this paper is as follows. In Section 2, we present some preliminaries in fractional calculus. In Section 3, BPs are introduced and then we approximate functions by using BPs and we show the properties of BPs by several Lemmas and corollaries. We make a new operational matrix for fractional derivative by BPs in Section 4. In Section 5, we apply BPs for solving time varying fractional optimal control problems. In Section 6, numerical examples are simulated to demonstrate the high performance of the proposed method. Finally, Section 7 concludes our work in this paper.

\section{Some preliminaries in fractional calculus}

In this section, we give some basic definitions and properties of the fractional calculus which are used further in this paper.

Definition 2.1. (See [5] ) We define

$$
\begin{aligned}
& C_{\mu}=\left\{f(t) \mid f(t)>0 \text { for } t>0 \text { and } f(t)=t^{p} f_{1}(t) \text { where } p>\mu \text { and } f_{1}(t) \in C[0, \infty)\right\}, \text { and } \\
& C_{\mu}^{n}=\left\{f(t) \mid f^{(n)}(t) \in C_{\mu}\right\} \text { where } n \in N, \mu \in R .
\end{aligned}
$$

Definition 2.2. (See [5]) The Riemann-Liouville fractional integral operator of order $\alpha \geq 0$, of a function $f \in C_{\mu}, \mu \geq-1$, is defined as

$$
\begin{aligned}
I^{\alpha} f(t) & =\frac{1}{\Gamma(\alpha)} \int_{0}^{t}(t-x)^{\alpha-1} f(x) d x, \quad \alpha>0, t>0, \\
I^{0} f(t) & =f(t),
\end{aligned}
$$

and for $n-1<\alpha \leq n, n \in \mathrm{N}, t>0, f \in C_{-1}^{n}$, the fractional derivative of $f(t)$ in the Caputo sense is defined as

$$
D^{\alpha} f(t)=I^{n-\alpha} D^{n} f(t)=\frac{1}{\Gamma(n-\alpha)} \int_{0}^{t}(t-x)^{n-\alpha-1} f^{(n)}(x) d x
$$

Property 2.3. (See [6-8]) For $f \in C_{\mu}, \mu \geq-1, \alpha, \beta \geq 0$ we have

$$
I^{\alpha} t^{\gamma}=\frac{\Gamma(\gamma+1)}{\Gamma(\alpha+\gamma+1)} t^{\alpha+\gamma}
$$

and for $n-1<\alpha \leq n, n \in \mathrm{N}$ and $f \in C_{\mu}^{n}, \mu \geq-1$ we see the following properties 
1. $D^{\alpha} I^{\alpha} f(t)=f(t)$,

2. $I^{\alpha} D^{\alpha} f(t)=f(t)-\sum_{k=0}^{n-1} f^{(k)}\left(0^{+}\right) \frac{x^{k}}{k !}, t>0$,

3. $D^{\beta} f(t)=I^{\alpha-\beta} D^{\alpha} f(t)$.

\section{Bernstein polynomials and their properties}

The Bernstein polynomials (BPs) of mth-degree are defined on the interval $[0,1]$ as follows:

$B_{i, m}(x)=\left(\begin{array}{l}m \\ i\end{array}\right) x^{i}(1-x)^{m-i}, \quad i=0,1, \cdots, m$

Corollary 3.1. Set $\left\{B_{0, m}(x), B_{1, m}(x), \cdots, B_{m, m}(x)\right\}$ is a complete basis in Hilbert space $L^{2}[0,1]$ and polynomials of degree $m$ can be expanded in terms of linear combination of $B_{i, m}(x)(i=0,1, \cdots, m)$ as follows

$P(x)=\sum_{i=0}^{m} c_{i} B_{i, m}(x)$.

Lemma 3.2. We can write $\Phi_{m}(x)=A T_{m}(x)$, where $A$ is a matrix upper triangular, $T_{m}(x)=\left[1, x, \ldots, x^{m}\right]^{T}$ and $\Phi_{m}(x)=\left[B_{0}(x), B_{2}(x), \ldots, B_{m}(x)\right]^{T}$.

Proof. Using binomial expansion of $(1-x)^{m-i}$, we have

$$
\begin{aligned}
B_{i, m}(x) & =\left(\begin{array}{l}
m \\
i
\end{array}\right) x^{i}(1-x)^{m-i}=\left(\begin{array}{l}
m \\
i
\end{array}\right) x^{i}\left(\sum_{k=0}^{m-i}(-1)^{k}\left(\begin{array}{l}
m \\
i
\end{array}\right)\left(\begin{array}{c}
m-i \\
k
\end{array}\right) x^{k}\right) \\
& =\sum_{k=0}^{m-i}(-1)^{k}\left(\begin{array}{l}
m \\
i
\end{array}\right)\left(\begin{array}{c}
m-i \\
k
\end{array}\right) x^{i+k}, i=0,1 \ldots, m .
\end{aligned}
$$

Therefore we can write

$\Phi_{m}(x)=A T_{m}(x)$,

where $A=\left(a_{i, j}\right)_{i, j=1}^{m+1}$ and $a_{i+1, j+1}= \begin{cases}(-1)^{j-i}\left(\begin{array}{l}m \\ i\end{array}\right)\left(\begin{array}{c}m-i \\ j-i\end{array}\right) & i \leq j, \quad i, j=0,1, \ldots, m . \\ 0 & i>j,\end{cases}$ 
Lemma 3.3. (see [9]) Let $L^{2}[0,1]$ be a Hilbert space with the inner product $<f, g>=\int_{0}^{1} f(x) g(x) d x$, and $y \in L^{2}[0,1]$. Then, we can find the unique vector $c=\left[c_{1}, c_{2}, \cdots, c_{m}\right]^{T}$ such that $y(x) \approx \sum_{i=0}^{m} c_{i} B_{i, m}(x)=c^{T} \Phi_{m}(x)$.

Corollary 3.4. In lemma 3.3, we have $c^{T}=<f, \Phi_{m}>Q^{-1}$, such that $<f, \Phi_{m}>=\int_{0}^{1} f(x) \Phi_{m}(x)^{T} d x=\left[<f, B_{0, m}>,<f, B_{1, m}>, \cdots,<f, B_{m, m}>\right]$ and $Q=\left(Q_{i, j}\right)_{i, j=1}^{m+1}$ is as follows

$$
Q_{i+1, j+1}=\int_{0}^{1} B_{i, m}(x) B_{j, m}(x) d x=\frac{\left(\begin{array}{l}
m \\
i
\end{array}\right)\left(\begin{array}{c}
m \\
j
\end{array}\right)}{(2 m+1)\left(\begin{array}{l}
2 m \\
i+j
\end{array}\right)}, i, j=0,1, \cdots m .
$$

Lemma 3.5. Suppose that $c_{(m+1) \times 1}$ is an arbitrary vector. The operational matrix of product $\hat{C}_{(m+1) \times(m+1)}$ using BPs can be given as follows:

$c^{T} \Phi_{m}(x) \Phi_{m}(x)^{T} \approx \Phi_{m}(x)^{T} \hat{C}$

Proof. From (12) we have

$$
\begin{aligned}
c^{T} \Phi_{m}(x) \Phi_{m}(x)^{T} & =c^{T} \Phi_{m}(x)\left(T_{m}(x)^{T} A^{T}\right)=\left[c^{T} \Phi_{m}(x), x\left(c^{T} \Phi_{m}(x)\right), \ldots, x^{m}\left(c^{T} \Phi_{m}(x)\right)\right] A^{T} \\
& =\left[\sum_{i=0}^{m} c_{i} B_{i, m}(x), \sum_{i=0}^{m} c_{i} x B_{i, m}(x), \ldots, \sum_{i=0}^{m} c_{i} x^{m} B_{i, m}(x)\right] A^{T} .
\end{aligned}
$$

Now, we approximate all functions $x^{k} B_{i, m}(x)$ in terms of $\Phi_{m}(x)$. Thus we define $e_{k, i}=\left[e_{k, i}^{0}, e_{k, i}^{1}, \ldots, e_{k, i}^{m}\right]^{T}$, then by (14) we can write $x^{k} B_{i, m}(x) \approx e_{k, i} \Phi_{m}(x), \quad i, k=0,1, \ldots, m$.

So, we get 


$$
\begin{aligned}
e_{k, i} & =Q^{-1}\left(\int_{0}^{1}\left(x^{k} B_{i, m}(x)\right) \Phi_{m}(x) d x\right) \\
& =Q^{-1}\left[\int_{0}^{1} x^{k} B_{i, m}(x) B_{0, m}(x) d x, \int_{0}^{1} x^{k} B_{i, m}(x) B_{1, m}(x) d x, \ldots, \int_{0}^{1} x^{k} B_{i, m}(x) B_{m, m}(x) d x\right]^{T} \\
& =\frac{Q^{-1}\left(\begin{array}{l}
m \\
i
\end{array}\right)}{2 m+k+1}\left[\frac{\left(\begin{array}{l}
m \\
0
\end{array}\right)}{\left(\begin{array}{l}
2 m+k \\
i+k
\end{array}\right)}, \frac{\left(\begin{array}{l}
m \\
1
\end{array}\right)}{\left(\begin{array}{l}
2 m+k \\
i+k+1
\end{array}\right)}, \ldots, \frac{\left(\begin{array}{l}
m \\
m
\end{array}\right)}{\left(\begin{array}{l}
2 m+k \\
i+k+m
\end{array}\right)}\right]^{T}, \quad i, k=0,1, \ldots, m .
\end{aligned}
$$

Then, we have

$$
\begin{aligned}
\sum_{i=0}^{m} c_{i} x^{k} B_{i, m}(x) & \approx \sum_{i=0}^{m} c_{i}\left(\sum_{j=0}^{m} e_{k, i}^{j} B_{j, m}(x)\right)=\sum_{i=0}^{m} B_{j, m}(x)\left(\sum_{i=0}^{m} c_{i} e_{k, i}^{j}\right) \\
& =\Phi_{m}(x)^{T}\left[\sum_{i=0}^{m} c_{i} e_{k, i}^{0}, \sum_{i=0}^{m} c_{i} e_{k, i}^{1}, \ldots, \sum_{i=0}^{m} c_{i} e_{k, i}^{m}\right]^{T} \\
& =\Phi_{m}(x)^{T}\left[e_{k, 0}, e_{k, 1}, \ldots, e_{k, m}\right] c=\Phi_{m}(x)^{T} V_{k+1} c
\end{aligned}
$$

where $V_{k+1}(k=0,1, \ldots m)$ is an $(m+1) \times(m+1)$ matrix that has vectors $e_{k, i}(i=0,1, \ldots, m)$ for each column's. If we define $\bar{C}=\left[V_{1} c, V_{2} c, \ldots, V_{m} c\right]$, then we get

$c^{T} \Phi_{m}(x) \Phi_{m}(x)^{T} \approx \Phi_{m}(x)^{T} \bar{C} A^{T}$,

and therefore we obtain the operational matrix of product $\hat{C}=\bar{C} A^{T}$.

\section{BPs operational matrix for fractional derivative}

In this section, we obtain the operational matrix for the fractional derivative. We can write

$D^{\alpha} \Phi_{m}(t)=\frac{1}{\Gamma(n-\alpha)} t^{n-\alpha-1} * \Phi_{m}^{(n)}(t), \quad 0 \leq t \leq 1$,

where $*$ denotes the convolution product.

By (12) we obtain

$$
D^{\alpha} \Phi_{m}(t)=\frac{1}{\Gamma(n-\alpha)} A\left(t^{n-\alpha-1} * T_{m}^{(n)}(t)\right)=A D^{\alpha} T_{m}(t)=A\left[D^{\alpha} 1, D^{\alpha} t, \ldots, D^{\alpha} t^{m}\right]^{T}
$$


where $\quad D^{\alpha} t^{j}= \begin{cases}0 & j=0, \ldots,\lceil\alpha\rceil-1, \\ \frac{\Gamma(j+1)}{\Gamma(j+1-\alpha)} t^{j-\alpha} & j=\lceil\alpha\rceil, \ldots, m .\end{cases}$

Therefore we have

$D^{\alpha} T_{m}(t)=\tilde{\Sigma} \tilde{T}$

where $\tilde{\Sigma}$ and $\tilde{T}$ are a $(m+1) \times(m+1)$ diagonal matrix and a $(m+1) \times 1$ matrix, respectively as follows:

$$
\tilde{\Sigma}=\left(\tilde{\Sigma}_{i, j}\right)_{i, j=1}^{m+1}, \quad \tilde{\Sigma}_{i+1, j+1}= \begin{cases}\frac{\Gamma(j+1)}{\Gamma(j+1-\alpha)} & i, j=\lceil\alpha\rceil, \ldots, m \text { and } i=j, \\ 0 & \text { otherwise }\end{cases}
$$

and $\tilde{T}=(\widetilde{T})_{i}=\left\{\begin{array}{ll}0 & i=0, \ldots,\lceil\alpha\rceil-1 \\ t^{i-\alpha} & i=\lceil\alpha\rceil, \ldots, m .\end{array}\right.$.

Now, we approximate $t^{i-\alpha}(i=\lceil\alpha\rceil, \ldots, m)$ with respect to BPs by using (13). Therefore, we can write

$t^{i-\alpha} \approx P_{i}^{T} \Phi_{m}(t)$,

where $P_{i}, \quad(i=\lceil\alpha\rceil, \ldots, m)$ is a vector $(m+1) \times 1$. So, we have

$$
\begin{aligned}
P_{i}=Q^{-1}\left(\int_{0}^{1} t^{i-\alpha} \Phi_{m}(t) d t\right) & =Q^{-1}\left[\int_{0}^{1} t^{i-\alpha} B_{0, m}(t) d t, \int_{0}^{1} t^{i-\alpha} B_{1, m}(t) d t, \ldots, \int_{0}^{1} t^{i-\alpha} B_{m, m}(t) d t\right]^{T} \\
& =Q^{-1} \bar{P}_{i},
\end{aligned}
$$

where

$$
\begin{aligned}
& \bar{P}_{i}=\left[\bar{P}_{i, 0}, \bar{P}_{i, 1}, \ldots, \bar{P}_{i, m}\right]^{T}, \\
& \bar{P}_{i, j}=\int_{0}^{1} t^{i-\alpha} B_{j, m}(t) d t=\frac{m ! \Gamma(i+j-\alpha+1)}{j ! \Gamma(i+m-\alpha+2)}, \quad i=\lceil\alpha\rceil, \ldots, m \text { and } \quad j=0,1, \ldots, m .
\end{aligned}
$$

Now, we suppose $P$ is an $(m+1) \times(m+1)$ matrix that has vector zero in $\lceil\alpha\rceil$ first column and vector $P_{i}$ in (i+1)th column's for $i=\lceil\alpha\rceil, \ldots, m$.

Finally, from (17)-(24), we obtain 


$$
D^{\alpha} \Phi_{m}(t) \approx D_{\alpha} \Phi_{m}(t)
$$

where

$$
D_{\alpha} \approx A \tilde{\Sigma} P^{T}
$$

is called the Bernstein polynomials operational matrix of fractional derivative.

\section{BPs for solving time varying fractional optimal control problems}

Using Lemma 3.3, we can approximate the functions as follows:

$$
\begin{aligned}
& x(t) \approx c^{T} \Phi_{m}(t), \\
& u(t) \approx b^{T} \Phi_{m}(t), \\
& a_{1}(t) \approx a_{1}^{T} \Phi_{m}(t), \\
& a_{2}(t) \approx a_{2}^{T} \Phi_{m}(t),
\end{aligned}
$$

where $c, b, a_{1}, a_{2} \in R^{(m+1) \times 1}$.

by (25) and (27) we can write

$$
D^{\alpha} x(t) \approx c^{T} D_{\alpha} \Phi_{m}(t)
$$

Therefore, the problem (1)-(3) reduce to the following problem:

$$
\text { Minimize } \quad \frac{1}{2} \int_{0}^{1} c^{T} \Phi_{m}(t) \Phi_{m}(t)^{T} c+b^{T} \Phi_{m}(t) \Phi_{m}(t)^{T} b d t
$$

subject to the dynamic constrain

$$
c^{T} D_{\alpha} \Phi_{m}(t)=a_{1}^{T} \Phi_{m}(t) \Phi_{m}(t)^{T} c+a_{2}^{T} \Phi_{m}(t) \Phi_{m}(t)^{T} b
$$

and the initial condition

$$
c^{T} \Phi_{m}(0)=x_{0}
$$

Now, using Corollary 3.4 for (32) we can write 


$$
\text { Minimize } \begin{aligned}
J(c, b) & =\frac{1}{2} c^{T}\left(\int_{0}^{1} \Phi_{m}(t) \Phi_{m}(t)^{T} d t\right) c+\frac{1}{2} b^{T}\left(\int_{0}^{1} \Phi_{m}(t) \Phi_{m}(t)^{T} d t\right) b . \\
& =\frac{1}{2} c^{T} Q c+\frac{1}{2} b^{T} Q b
\end{aligned} .
$$

Also by Lemma 3.5 for (33) we have

$$
c^{T} D_{\alpha} \Phi_{m}(t)=\Phi_{m}(t)^{T} \hat{A}_{1} c+\Phi_{m}(t)^{T} \hat{A}_{2} b
$$

Now, by using tau method [10] we can generate algebraic equations from (36) as follows

$$
G_{j}(c, b)=\int_{0}^{1}\left(c^{T} D_{\alpha}-c^{T} \hat{A}_{1}^{T}-b^{T} \hat{A}_{2}^{T}\right) \Phi_{m}(t) B_{j, m}(t) d t=0, \quad j=0, \ldots, m-1,
$$

and from (34) we set $G_{m}=c^{T} \Phi_{m}(0)-x_{0}$.

Finally, the problem (1)-(3) has been reduced to a parameter optimization problem which can be stated as follows:

Find $c$ and $b$ which

$$
\text { Minimize } \quad J(c, b)=\frac{1}{2} c^{T} Q c+\frac{1}{2} b^{T} Q b,
$$

subject to the system of algebraic equations

$$
G_{j}(c, b)=0, \quad j=0, \ldots, m .
$$

For solving the above problem we use the Lagrange multipliers method. So, we define Lagrange function for the problem (38) and (39) as follows:

$L(c, b, \lambda)=\frac{1}{2} c^{T} Q c+\frac{1}{2} b^{T} Q b+\sum_{j=0}^{m} \lambda_{j} G_{j}(c, b)$,

where $\lambda=\left[\lambda_{0}, \ldots, \lambda_{m}\right]^{T}$ is the unknown Lagrange multiplier. Now, we consider the necessary conditions for the extremum and obtain the following systems of algebraic equations

$$
\begin{aligned}
& \frac{\partial L}{\partial c}=0 \\
& \frac{\partial L}{\partial b}=0 \\
& \frac{\partial L}{\partial \lambda}=0 .
\end{aligned}
$$


Equations (41)-(43) can be solved for $c, b$ and $\lambda$ by Newton's iterative method. Then, we get the approximate value of the state functions $x(t)$ and the control functions $u(t)$ from (27) and (28), respectively.

\section{Numerical examples}

To demonstrate the applicability and to validate the numerical scheme, we apply the present method for the following examples.

Example 1. Consider the following time invariant problem $[1,2]$

Minimize $\quad J=\frac{1}{2} \int_{0}^{1} x^{2}(t)+u^{2}(t) d t$

subject to the system dynamics

$D^{\alpha} x(t)=-x(t)+u(t)$

with initial condition

$x(0)=1$.

For this problem we have the exact solution in the case of $\alpha=1$ as follows

$x(t)=\cosh (\sqrt{2} t)+\beta \sinh (\sqrt{2} t)$,

$u(t)=(1+\sqrt{2} \beta) \cosh (\sqrt{2} t)+(\sqrt{2}+\beta) \sinh (\sqrt{2} t)$,

where $\beta=-\frac{\cosh (\sqrt{2})+\sqrt{2} \sinh (\sqrt{2})}{\sqrt{2} \cosh (\sqrt{2})+\sinh (\sqrt{2})}$.

Figures 1 and 2 show the state and the control variables, respectively, as a function of time for $m=5$, for different values of $\alpha$. These figures show that as $\alpha$ approaches close to 1 , the numerical solutions for both the state and the control variables approach to the analytical solutions for $\alpha=1$ as expected. In Figures 1 and 2, only the numerical results for $\alpha=1$ are presented. This is because, for $\alpha=1$ the analytical and the numerical results overlap. In Figs. 3 and 4, we see the absolute error of obtained results for $m=10$ and $\alpha=1$. Also, in Table 1 and 2, the absolute error of $x(t)$ for when $\alpha=1$ and $m=4,5$ is demonstrated and is compared with [1]. 


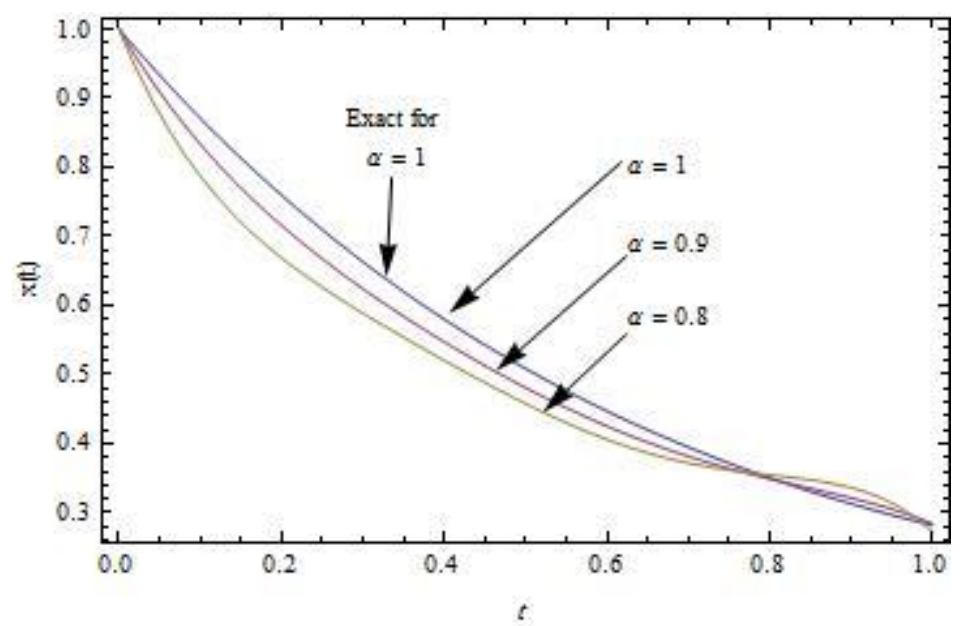

Fig 1. Approximate solutions of $x(t)$ for $m=5$ and different values of $\alpha$ and exact solution for $\alpha=1$ in example 1 .

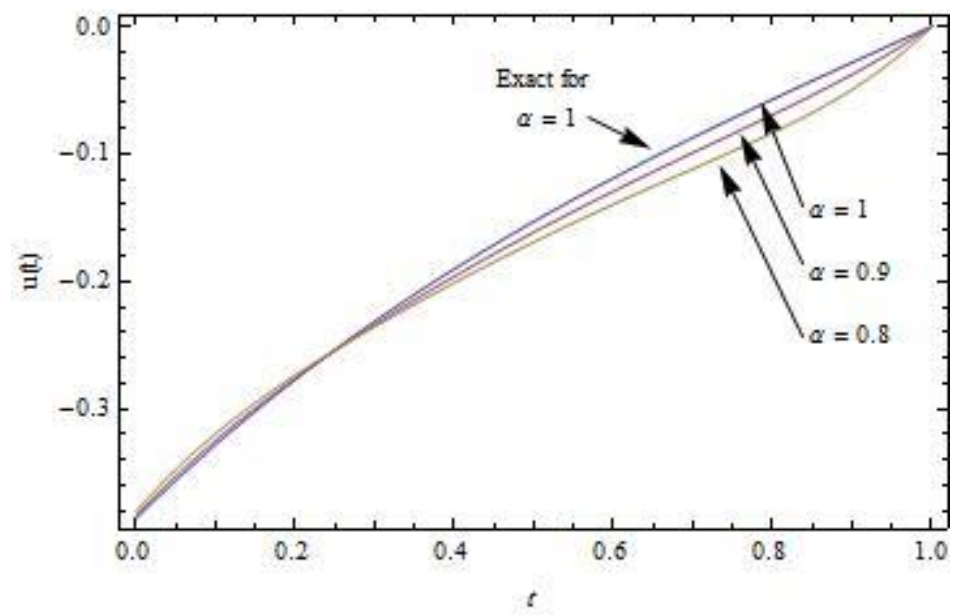

Fig 2. Approximate solutions of $u(t)$ for $m=5$ and different values of $\alpha$ and exact solution for $\alpha=1$ in example 1 .

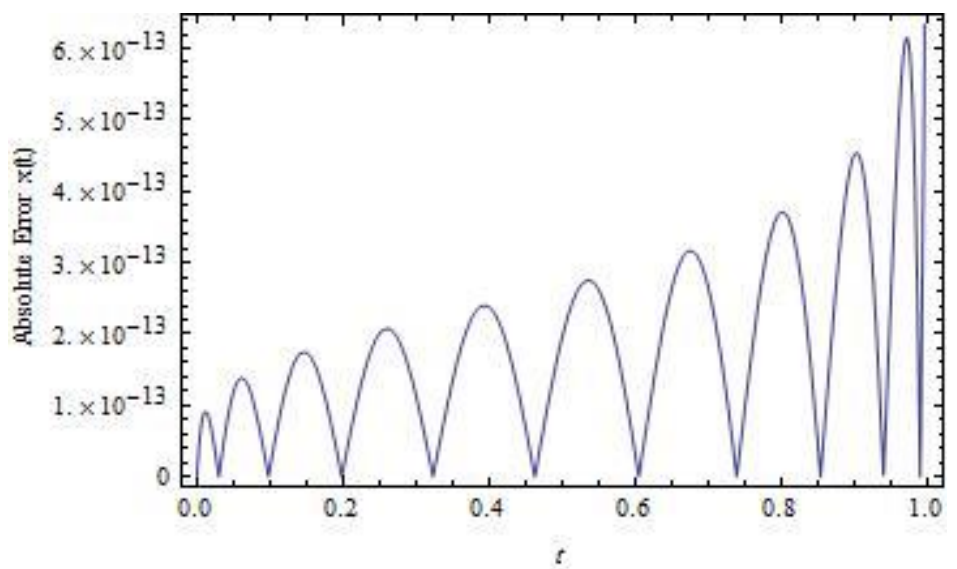

Fig 3. Plot of absolute error function $x(t)$ for $\alpha=1$ and $m=10$ in example 1 . 


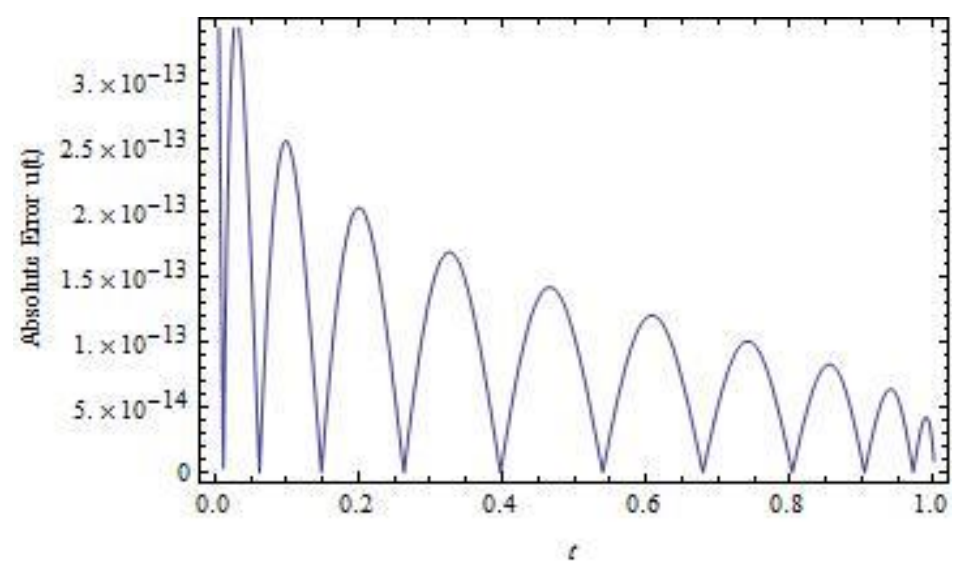

Fig 4. Plot of absolute error function $u(t)$ for $\alpha=1$ and $m=10$ in example 1 .

Table 1. Absolute error $x(t)$ for $\alpha=1, m=4$ and different values $t$ in example 1 .

\begin{tabular}{|c|c|c|}
\hline$t$ & $m=4[1]$ & Present Method $m=4$ \\
\hline 0 & 0.0000899 & 0 \\
0.1 & 0.0000477 & 0.00001560360486785317 \\
0.2 & 0.0000325 & 0.000026407578959108946 \\
0.3 & 0.0000774 & 0.000036101485012540735 \\
0.4 & 0.0000213 & $3.899031830290056 \times 10^{-6}$ \\
0.5 & 0.0000643 & 0.00003810091250144865 \\
0.6 & 0.000103 & 0.00004946576317460538 \\
0.7 & 0.000112 & 0.000010110645709493493 \\
0.8 & 0.0000914 & 0.000055108397533421005 \\
0.9 & 0.0000941 & 0.00005539673693399916 \\
\hline
\end{tabular}

Table 2. Absolute error $x(t)$ for $\alpha=1, m=5$ and different values $t$ in example 1 .

\begin{tabular}{|c|c|c|}
\hline$t$ & {$[1] m=5$} & Present Method $m=5$ \\
\hline 0 & 0.00000625 & 0 \\
0.1 & 0.0000134 & $1.2376247904288817 \times 10^{-7}$ \\
0.2 & 0.0000212 & $2.1260006768386575 \times 10^{-6}$ \\
0.3 & 0.0000324 & $1.787127701380342 \times 10^{-8}$ \\
0.4 & 0.0000473 & $2.6023598275193294 \times 10^{-6}$ \\
0.5 & 0.0000620 & $1.8611311257688001 \times 10^{-6}$ \\
0.6 & 0.0000749 & $1.6945524689004898 \times 10^{-6}$ \\
0.7 & 0.0000888 & $3.600201665232561 \times 10^{-6}$ \\
0.8 & 0.000107 & $1.4930994740591785 \times 10^{-7}$ \\
0.9 & 0.000131 & $5.091619992403373 \times 10^{-6}$ \\
\hline
\end{tabular}


Example 2. This example considers a time varying fractional optimal control problem [1, 2]. Find the control $u(t)$ which minimizes the performance index $J$ given in Example 1 subject to the following dynamical system

$D^{\alpha} x(t)=t x(t)+u(t)$

with initial condition

$x(0)=1$

Figs. 5 and 6 show the state $x(t)$ and the control $u(t)$ as functions of $t$ for different values of $\alpha$. These figures show that as $\alpha$ approaches close to 1 , the numerical solutions for both the state and the control variables approach to the solutions for $\alpha=1$ as expected. The numerical solution obtained with the proposed method for fractional orders of $\alpha$ matches those found in the literature.

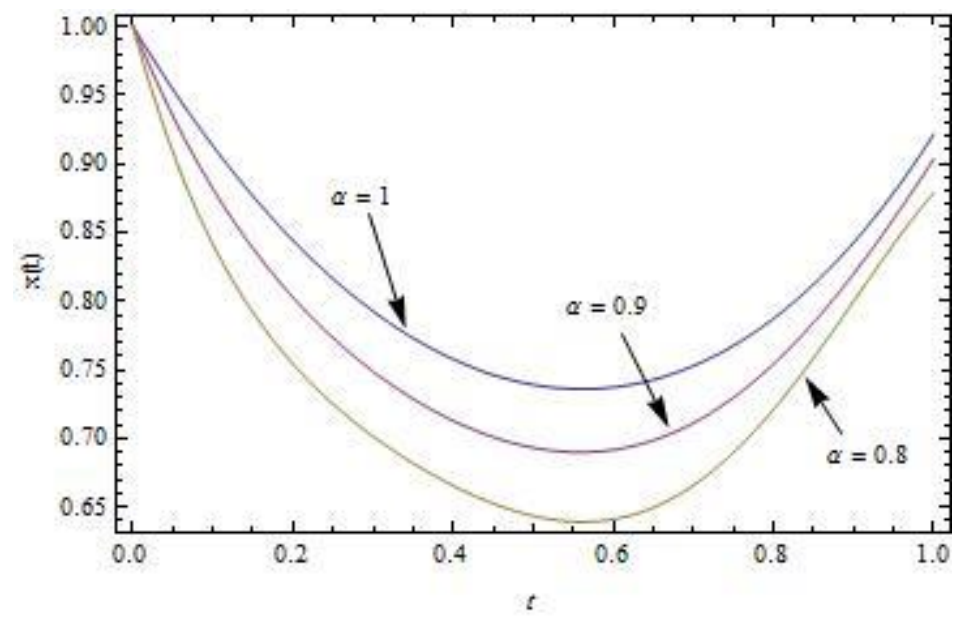

Fig 5. Approximate solutions of $x(t)$ for $m=5$ and different values of $\alpha$ in example 2 .

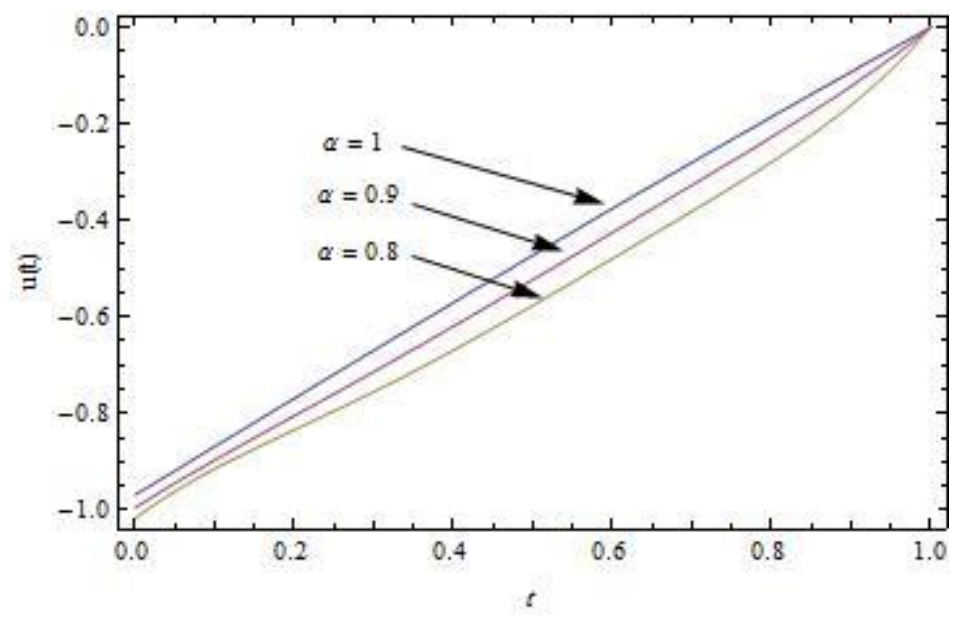

Fig 6. Approximate solutions of $u(t)$ for $m=5$ and different values of $\alpha$ in example 2 . 


\section{Conclusion}

In this work, by Bernstein polynomials we obtained operational matrices of the product and fractional derivative. Then we reduced the time varying fractional optimal control problem to a system of algebraic equations that can be solved easily. We saw that the obtained results in examples were in good agreement with the exact solution and approximate solution of other methods. Also, we observed that the solutions for the fractional optimal control problems approach to the solutions for standard optimal control problems as the order of the fractional derivative approaches to 1.

\section{Reference:}

[1] A. Lotfi, M. Dehghan, S.A. Yousefi, A numerical technique for solving fractional optimal control problems, Computers and Mathematics with Applications 62 (2011) 1055-1067.

[2] O.P. Agrawal, A general formulation and solution scheme for fractional optimal control problems, Nonlinear Dynam. 38 (2004) 323-337.

[3] O.P. Agrawal, D. Baleanu, A Hamiltonian formulation and a direct numerical scheme for fractional optimal control problems, J. Vib. Control 13 (2007) 1269-1281.

[4] D. Baleanu, O. Defterli, O.P. Agrawal, A central difference numerical scheme for fractional optimal control problems, J. Vib. Control 15 (4) (2009) 583-597.

[5] M. Caputo, Linear models of dissipation whose Q is almost frequency independent II, Geophys. J. Roy. Astronom. Soc. 13 (1967) 529-539.

[6] Y. Luchko and R. Gorneflo, The initial value problem for some fractional differential equations with the Caputo derivative, Preprint series A08-98, Fachbereich Mathematik und Informatik, Freie Universitat Berlin, (1998).

[7] K. S. Miller, B. Ross, An Introduction to the Fractional Calculus and Fractional Differential Equations, John Wiley and Sons, Inc., New York, (1993).

[8] K.B. Oldham and J. Spanier, The Fractional Calculus, Academic Press, New York, (1974).

[9] E. Kreyszig, Introduction Functional Analysis with Applications, John Wiley and Sons Incorporated, (1978).

[10] C. Canuto, M.Y. Hussaini, A. Quarteroni, T.A. Zang, Spectral Methods in Fluid Dynamic, Prentice-Hall, Englewood Cliffs, NJ, (1988). 